\title{
A PROPERTY OF FINITE SIMPLE NON-ABELIAN GROUPS
}

\author{
W. D. MAURER AND JOHN L. RHODES ${ }^{1}$
}

It has been proved by Fröhlich [1] that the collection of inner automorphisms of a finite simple non-abelian group $G$ generates the group (under pointwise multiplication) of all functions from $G$ into $G$ leaving the identity fixed, and, conversely, that the only finite groups with this property are the simple non-abelian groups, $Z_{2}$, and $[e]$. It follows directly that the inner automorphisms and the constant functions of a simple non-abelian group into itself generate the entire group of functions from $G$ into $G$. The purpose of this note is to prove the following generalization of this theorem.

TheOREM. Let $G$ be a finite group of order $\mathrm{g} \geqq 3$, and let $A$ be a finite set of order $a \geqq 3$. Let $F(A, G)$ be the group of all functions $f: A \rightarrow G$ under the operation $\left(f_{1} \cdot f_{2}\right)(a)=f_{1}(a) \cdot f_{2}(a)$, and let $H$ be a subgroup of $F(A, G)$ such that:

(a) $f^{\circ} \in H$, where $f^{\circ}(a)=g$, all $a \in A$, for each $g \in G$;

(b) if $x, y \in A$, there exists $f \in H$ with $f(x) \neq f(y)$.

Then $H$ is necessarily equal to $F(A, G)$ if and only if $G$ is simple nonabelian.

Proof. Let $G$ be simple non-abelian. A $j$-tuple $\left(g_{1}, \cdots, g_{j}\right)$ of elements $g_{i} \in G, j \leqq \mathfrak{a}$, is called accessible if for every $j$-tuple $\left(a_{1}, \cdots, a_{j}\right)$ of distinct members of $A$ there exists $f \in H$ such that $f\left(a_{i}\right)=g_{i}$, $1 \leqq i \leqq j$. We prove all $j$-tuples (in particular, all $a$-tuples) accessible by induction on $j$. All 1-tuples are accessible, by (a) above. It suffices to show that all $j$-tuples of the form $(g, e, \cdots, e)$ are accessible, since $H$ is a subgroup. Let $\alpha=\left(a_{1}, \cdots, a_{j}\right)$ be a particular $j$-tuple of distinct members of $A$, and let $G_{\alpha} \subseteq G$ be the set of all $g \in G$ for which there exists $f \in H, f\left(a_{1}\right)=g, f\left(a_{i}\right)=e, 2 \leqq i \leqq j$; since $H$ is a subgroup of $F(A, G), G_{\alpha}$ is a subgroup of $G$, and, in fact, it is seen to be a normal subgroup by considering $f^{x} \cdot f \cdot f^{x^{-1}}$. Since $G$ is simple, it suffices to show that $G_{\alpha} \neq[e]$. For $j=2$, this follows from (b) above; if $f\left(a_{1}\right)$ $\neq f\left(a_{2}\right)$, then $f^{\prime}=f \cdot f^{z}$, where $z=\left(f\left(a_{2}\right)\right)^{-1}$, satisfies $f^{\prime}\left(a_{1}\right) \neq e, f^{\prime}\left(a_{2}\right)=e$. Now assume $j \geqq 3$. Let $g_{1}, g_{2} \in G$ be such that $g_{1} g_{2} \neq g_{2} g_{1}$. Such elements exist because $G$ is non-abelian. Since all $(j-1)$-tuples are accessible, there exist $f_{1}, f_{2} \in H$ such that the values of $f_{1}$ and $f_{2}$ on the elements

Received by the editors February 12, 1963 and, in revised form, January 3, 1964.

1 The second-named author proved the corollary presented here by a somewhat complicated method assuming [1]. The first author obtained the present proof. 
$a_{i}$ are given by the following diagram:

$$
\begin{array}{rlllll}
a & =a_{1} & a_{2} & a_{3} & a_{4} \cdots a_{n} \\
f_{1}(a) & =g_{1} & e & x & e & e \\
f_{2}(a) & =g_{2} & y & e & e & e
\end{array}
$$

where $x$ and $y$ are (unspecified) elements of $G$. Then the values of $f_{3}=f_{1}^{-1} \cdot f_{2}^{-1} \cdot f_{1} \cdot f_{2}$ on the elements $a_{i}$ are given by

$$
\begin{aligned}
& a=a_{1} \quad a_{2} \quad a_{3} \quad a_{4} \cdots a_{n} \\
& f_{3}(a)=\left[g_{1} g_{2}\right] \quad e \quad e \quad e \quad e,
\end{aligned}
$$

where $\left[g_{1} g_{2}\right]=g_{1}^{-1} g_{2}^{-1} g_{1} g_{2}$, which shows that $\left[g_{1} g_{2}\right] \in G_{\alpha}$ and, therefore, $G_{\alpha} \neq[e]$.

Conversely, suppose that $G$ is either nonsimple or abelian. If $G$ has a proper nontrivial normal subgroup $N$, then

$$
H=\left\{f: A \rightarrow G \mid f(x) f(y)^{-1} \in N, \text { all } x, y \in A\right\}
$$

satisfies the requirements (a) and (b) of the theorem, and is a proper subset of $F(A, G)$. To prove $H$ a subgroup, we note that if $f(x) f(y)^{-1}$ $=h \in N, g(x) g(y)^{-1}=k \in N$, then

$$
\begin{aligned}
f(x) g(x)(f(y) g(y))^{-1} & =f(x)\left(g(x) g(y)^{-1}\right) f(y)^{-1}=f(x) k f(y)^{-1} \\
& =f(x) k f(x)^{-1} f(x) f(y)^{-1}=f(x) k f(x)^{-1} h,
\end{aligned}
$$

and this is in $N$ since $f(x) k f(x)^{-1}$ is a conjugate of $k$.

If $G$ is abelian, then the order of the product of two subgroups of the abelian group $F(A, G)$ is less than or equal to the product of the orders. Hence, let $\mathfrak{a} \leqq \mathfrak{g}$, let $G_{1}$ be the subgroup consisting of the functions $f^{\circ}$, and let $G_{2}$ be the cyclic subgroup generated by any function $f$ such that $f\left(a_{1}\right) \neq f\left(a_{2}\right)$ for all $a_{1}, a_{2} \in A$. Then we may set $H=G_{1} \cdot G_{2}$; the order of $G_{1}$ is $\mathfrak{g}$, and the order of $G_{2}$ is not greater than $\mathfrak{g}$, since $\mathrm{g}_{i}^{\mathrm{g}}=e$ for all $\mathrm{g}_{i} \in G$. Thus the order of $H$ is not greater than $\mathrm{g}^{2}$, whereas the order of $F(A, G)$ is $\mathfrak{g}^{\mathfrak{a}}, \mathfrak{a} \geqq 3$. If $\mathfrak{a}>\mathfrak{g}, A^{\prime} \subset A$ is of order $\mathfrak{g} \geqq 3$, and $H^{\prime} \subset F\left(A^{\prime}, G\right)$ is a proper subgroup satisfying conditions (a) and (b) of the theorem, then the set $H$ of functions whose restrictions to $A^{\prime}$ are in $H^{\prime}$ also satisfies conditions (a) and (b) and is also a proper subgroup. This completes the proof of the theorem.

If we set $A=G$, we obtain that the constant functions and the identity inner automorphism (which certainly satisfies condition (b) of the theorem) of a simple non-abelian group generate the entire group of functions from $G$ into $G$. This generalizes Fröhlich's theorem mentioned above; in fact, from this, Fröhlich's theorem follows immediately. The collection $G_{K}$ of constant functions on $G$ normalizes 
the group $G_{I}$ of pointwise products of inner automorphisms of $G$. If $G_{1}$ is the set of all functions on $G$ fixing the identity, then $G_{I}=G_{1}$ if and only if the group generated by $G_{I}$ and $G_{K}$ is $F(G, G)$. Thus, if $G$ is simple non-abelian, $G_{I}=G_{1}$.

If we set $A=G^{n}$, the direct product of $G$ taken with itself $n$ times, we obtain a result useful in the algebraic theory of machines. Let $X_{n}$ be a set of $n$ elements, and let $a_{1}, \cdots, a_{k}$ be elements of $G \cup X_{n}$ (disjoint union) for some finite group $G$. Then the product function of $n$ variables mapping from $G^{n}$ into $G$ associated with $\left(a_{1}, \cdots, a_{k}\right)$ is defined as follows. If $X_{n}=\left[x_{1}, \cdots, x_{n}\right]$, then $f\left(g_{1}, \cdots, g_{n}\right)=b_{1} \cdots b_{k}$, where

$$
\begin{array}{ll}
b_{i}=a_{i} & \text { for } a_{i} \in G, \\
b_{i}=g_{j} & \text { for } a_{i}=x_{j} \in X_{n} .
\end{array}
$$

Thus we may associate a product function $f: G^{n} \rightarrow G$ with each sequence of elements of $G \cup X_{n}$. This correspondence is a homomorphism of the free semigroup of finite sequences of elements of $G \cup X_{n}$ into the group of functions from $G^{n}$ into $G$ under pointwise multiplication. Its image, as a subsemigroup of a finite group, is itself a group $F_{n}(G)$. Now the following result holds.

Corollary. Every function $f: G^{n} \rightarrow G, n \geqq 1$, belongs to $F_{n}(G)$ if and only if $G$ is a simple non-abelian group (or $G=[e]$ ).

Proof. For $G=[e]$ the theorem is obvious. It is easy to verify that $F_{1}(G)$ is generated by the inner automorphisms and the constant functions. Thus if $F_{1}(G)$ consists of all functions we have seen that $G_{I}$, the group of pointwise products of inner automorphism, consists of all functions fixing the identity and thus $G$ can possess no proper nontrivial subgroups. In this situation $G$ is simple non-abelian or $G=Z_{p}$, the integers modulo the prime $p$. However, $F_{n}\left(Z_{p}\right)$ is seen to have order strictly less than the order of $F\left(Z_{p}^{n}, Z_{p}\right)$ for all $n \geqq 2$ by performing a trivial calculation.

Now let $G$ be simple non-abelian. The constant functions belong to $F_{n}(G)$ since they correspond to the sequences $\left(g_{i}\right)$ of length 1 . If $\left(g_{1}, \cdots, g_{n}\right)$ and $\left(g_{1}^{\prime}, \cdots, g_{n}^{\prime}\right)$ are distinct elements of $G^{n}$, there exists an index $i$ for which $g_{i} \neq g_{i}^{\prime}$, and the product function corresponding to the sequence $\left(x_{i}\right)$ of length 1 satisfies condition (b) for this pair of elements of $G^{n}$. The corollary now follows immediately from the theorem.

\section{BIBLIOGRAPHY}

1. A. Fröhlich, The near-ring generated by the inner automorphisms of a finite simple group, J. London Math. Soc. 33 (1958), 95-107.

University of California, Berkeley 